\title{
Rieger's syndrome with pericentric inversion of chromosome 6
}

\author{
MURK-HEIN HEINEMANN, ROY BREG, AND EDWARD COTLIER \\ From the Department of Ophthalmology and Visual Science and Institute of Human Genetics, \\ Yale University School of Medicine, New Haven, Connecticut, USA
}

SUMMARY Pericentric inversion of chromosome $6(6 \mathrm{p}+\mathrm{q}-)$ was found in a girl with Rieger's syndrome and in her father. The only ocular signs in the father were prominent iris mounds and Schwalbe's line. The association of chromosomal anomalies with Rieger's syndrome indicates the need for a chromosome banding test in familial or sporadic patients with the syndrome and in patients with mild anomalies of the anterior chamber angle.

Rieger's syndrome is characterised by mesodermal anomalies of the anterior chamber angle and facial, dental, and musculoskeletal malformations. Major ocular abnormalities include hypoplasia of the anterior iris stroma, a prominent Schwalbe's line (posterior embryotoxon), and large peripheral anterior synechiae stretching from the peripheral and mid portions of the iris (Waring et al., 1975). Abnormalities of the iris such as polycoria and dyscoria and secondary corneal anomalies such as microcornea and macrocornea occur frequently (Waring et al., 1975). On the basis of an extensive review of 175 patients Alkemade (1969) concluded that Rieger's anomaly is inherited in an autosomal dominant pattern in $70 \%$ of cases, while $30 \%$ are thought to arise as isolated events (Alkemade, 1969). Thus, spontaneous or transmitted mutations associated with chromosomal anomalies may occur in Rieger's syndrome.

Tabbara et al. (1973) reported the case of a young man with Rieger's syndrome associated with an extra metacentric chromosome. A case of Rieger's syndrome with associated inverted chromosome 6 in a young girl is presented here. Similar chromosomal anomalies were found in the father, who presented minimal mesodermal anomalies of the anterior chamber angle.

\section{Case report}

CASE 1

The proposita is a 10-year-old white girl who is the product of a 38-week gestation and uncomplicated

Address for reprints: Edward Cotlier, MD, Yale University School of Medicine, Department of Ophthalmology and Visual Science, 333 Cedar Street, New Haven, Connecticut 06510, USA vaginal delivery. The birth weight was $3.335 \mathrm{~kg}$. The only physical abnormality noted during the initial physical examination was epithelisation of the umbilicus. Soon after her birth hypoplastic upper gums were noted as well as a short lingual frenulum. She underwent frenulectomy at the age of 4 months.

The patient sat at 7 months of age and walked at 14 months despite bilateral tibial distortion (genu varus) which necessitated the wearing of a night brace for a brief period. She began to talk at 18 months. The child's height and weight were consistently within the 5th and 15 th percentiles, and she was evaluated for the possible presence of a growth hormone disturbance. Post-exercise levels of human growth hormone were $9 \mathrm{ng} / \mathrm{ml}$, near the lower limit of normal. Radiological appearances of the sella turcica were within normal limits. Bone age measurements using the standards of Greulick and Pyle showed no evidence for retarded skeletal maturation.

Her dentition has been markedly abnormal. At agc 6 months she was found to have hypoplasia of the maxilla. Mandibular incisors appeared at 7 months of age. Recent dental films disclosed no permanent teeth except for 6-year molars.

At 5 months of age unusually shaped pupils were noted. Eye examination revealed microcornea and diagonal pupils bilaterally. Phenylephrine $2.5 \%$ eye drops were prescribed for pupillary dilatation, but therapy was unsuccessful. An examination at 16 months of age showed intraocular pressures of $16 \mathrm{mmHg}$ bilaterally, and corneal diameters of $9.5 \mathrm{~mm}$ (horizontal) by $9.5 \mathrm{~mm}$ (vertical) in the right eye and $9.0 \mathrm{~mm}$ (horizontal) by $9.5 \mathrm{~mm}$ (vertical) in the left. When the child was $2 \frac{1}{2}$ years of age she was found to have an abnormally thickened and centrally displaced ridge of Schwalbe's line as well as numerous iris processes attached anteriorly to 


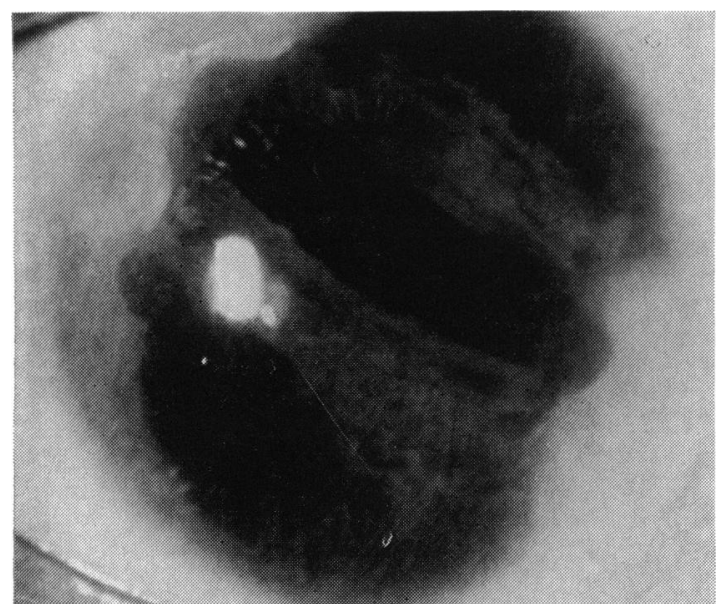

Fig. 1 Slit-lamp photograph of left eye of proband. Note the ill-defined limbus, dyscoria, ectropion uveae, and multiple defects in the abnormal iris stroma

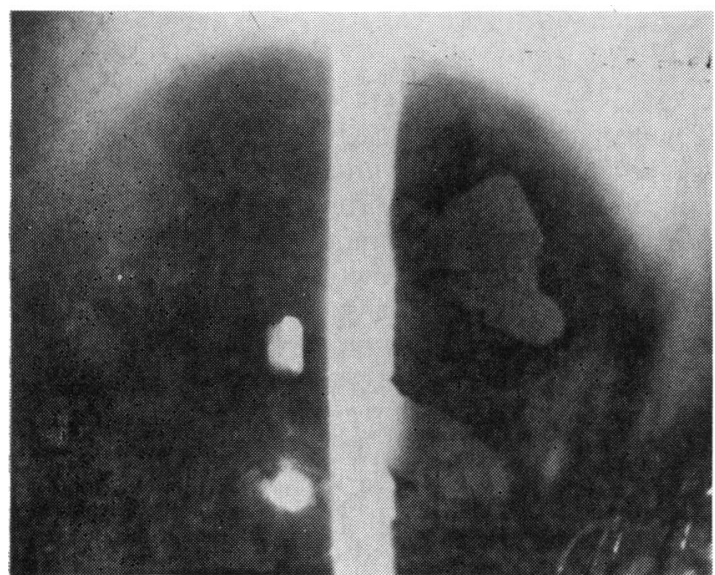

Fig. 2 Slit-lamp photograph of right eye of proband. Note the ill-defined limbus, dyscoric pupil, and multiple defects in the hypoplastic iris stroma

Schwalbe's line bilaterally, causing practically complete closure of the angle structures (Figs. 1 and 2). The irides were atrophic, with hypoplasia of the anterior stroma. Ophthalmoscopic examination showed normal fundi without abnormal cupping of the optic discs. Intraocular pressures were $16 \mathrm{mmHg}$ in both eyes and outflow facilities $\mathrm{C}=0.25$ bilaterally.

During this period the child was repeatedly examined under anaesthesia for evidence of glaucoma. There was one episode of intraocular pressure of $50 \mathrm{mmHg}$ bilaterally, which was treated with pilocarpine $4 \% 4$ times daily and acetazolamide 60 mg 4 times daily. Repeat tonometry during the following weeks gave normal pressures both off and on medication, and further drug therapy was discontinued. Dental examination showed maxillary hypoplasia and microdontia (Fig. 3).

At the age of 10 years the patient's visual acuity was $20 / 50$ with +2.00 to $6.00 \times 160^{\circ}$ right eye, and $20 / 30$ with +4.00 to $1.50 \times 150^{\circ}$ left eye. There was mild retraction and recession of the upper lip. Lids, orbits, and lacrimal apparatus were normal bilaterally. The range of extraocular muscle movement was full, with orthophoria at $20 \mathrm{ft}(6 \mathrm{~m})$ and 14 in $(36 \mathrm{~cm})$. Slit-lamp examination showed normal corneas but reduced depth of anterior chamber in both eyes. Pupils were elongated in a slit-like configuration, and large defects in the atrophic iris stroma were present.

Applanation tonometry gave intraocular pressures of $24 \mathrm{mmHg}$ in the right eye, and $16 \mathrm{mmHg}$ in the left. Gonioscopy of the right eye showed two small areas of open angle, superiorly and inferiorly. Schwalbe's line was abnormally thickened, and numerous iris processes were noted. The remainder of the angle was closed by peripheral anterior synechiae. The left eye had an anterior chamber angle very much like that of the right eye with many fine iris processes inserted anteriorly on to the prominent Schwalbe's line.

Goldmann perimetry showed full visual fields to 1 to 2,1 to 3 , and 1 to 4 test targets bilaterally. On ophthalmoscopic examination discs, vessels, maculae, and peripheral retinas were seen to be normal on both sides.

Karyotypes prepared from peripheral blood cultures and banded with Giemsa showed a modal

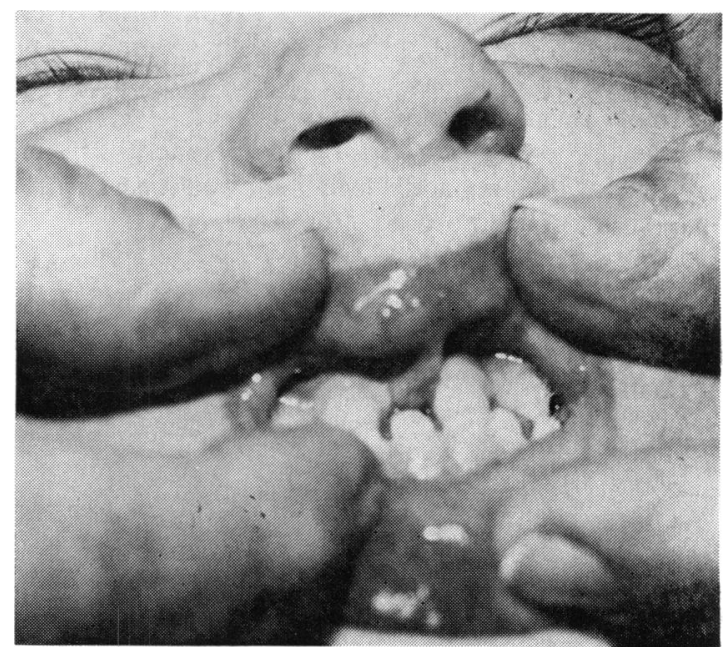

Fig. 3 Photograph of proband's teeth showing oligodontia and microdontia 


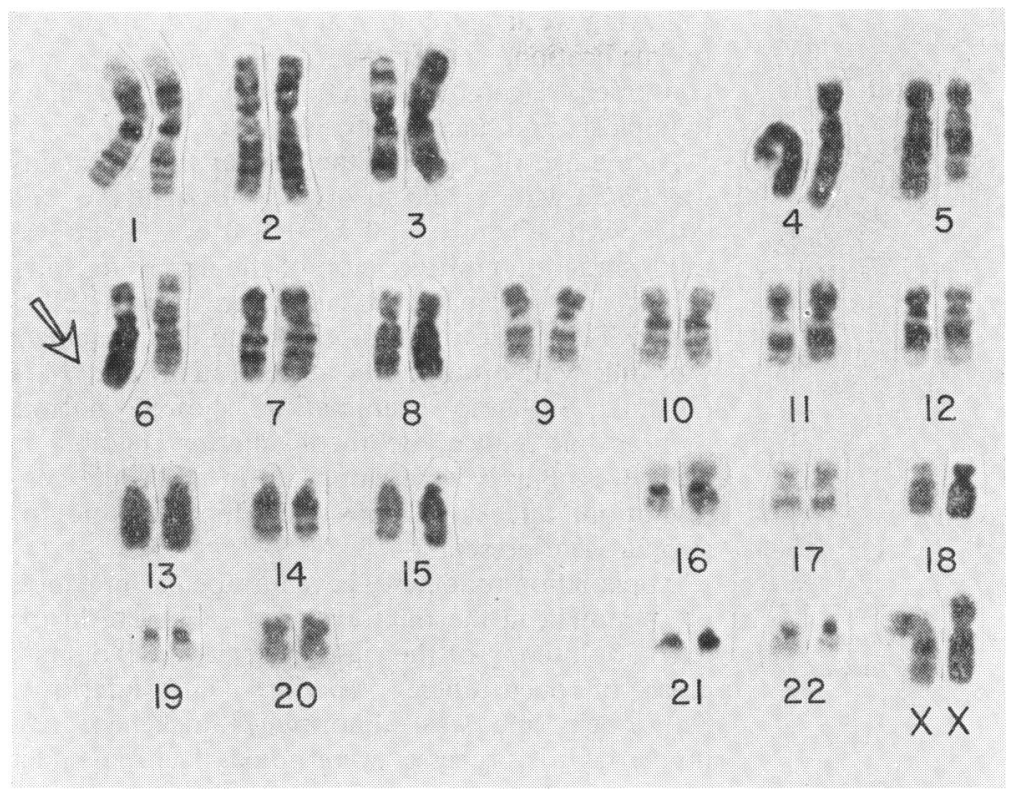

Fig. 4 Karyotype of proband

count of 46 with pericentric inversion of chromosome $6\left(6 \mathrm{p}+\mathrm{q}^{-}\right)$(Figs. 4 and 5$)$.

\section{CASE 2}

The father of the proband is 36 years old. Visual acuity was 20/20 right eye, 20/25 left eye, without correction. External examination showed the lids, orbits, and lacrimal apparatus to be within normal limits. Slit-lamp examination revealed normal conjunctivae and corneas. Gonioscopy was remarkable for prominent Schwalbe's line and iris mounds bilaterally. On ophthalmoscopic examination discs, vessels, maculae, and peripheral retinas were seen to be normal on both sides. Intraocular pressures were $17 \mathrm{mmHg}$ right eye and $15 \mathrm{mmHg}$ left eye.

Karyotypes prepared from peripheral blood cultures of the proband's father showed a pericentric inversion of chromosome $6(6 \mathrm{p}+\mathrm{q}-)$.

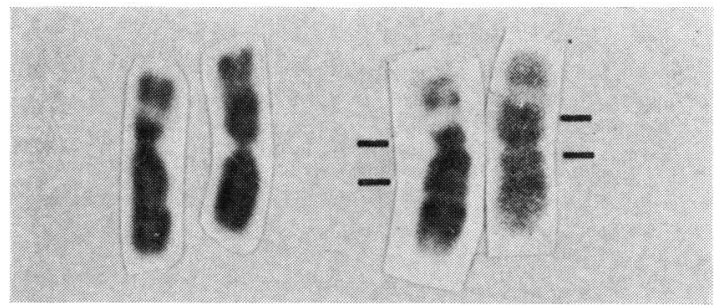

Fig. 5 To right (between lines) chromosome 6 pair from proband showing pericentric inversion. To left, chromosome 6 pair of father with pericentric inversion

\section{CASE 3}

The proband's mother is 33 years old. Visual acuity was $20 / 20$ bilaterally without correction. External appearances of the lids, orbits, and lacrimal apparatus were within normal limits. Slit-lamp examination revealed normal conjunctivae, corneas, anterior chambers, irides, and lenses. Gonioscopy revealed normal angle structures bilaterally. On ophthalmoscopic examination optic discs, vessels, maculae, and peripheral retinas were seen to be normal on both sides. Intraocular pressures were $14 \mathrm{mmHg}$ right eye and $14 \mathrm{mmHg}$ left eye.

Karyotypes prepared from peripheral blood cultures of the proband's mother were normal (46 XX).

\section{Discussion}

Pericentric inversions of autosomal chromosomes are rare events. Reviews of chromosomal analyses of large series of consecutive births have shown an incidence ranging from 0.16 to 0.56 per 1000 live births (Friedrich and Nielson, 1973). Ocular anomalies associated with pericentric inversions have been summarised by Jay (1977). Simple and typical colobomata of the iris have been found in 4 cases of pericentric inversions (Lele et al., 1965; Schmid, 1967; Mikelsaar et al., 1970). Pitt (1967) presented a case of an inversion of a C-group chromosome with associated exotropia, epicanthal folds, and hypertelorism; however, no anomalies of the iris or anterior chamber angle were noted. 
The highly variable constellation of ocular and systemic defects which have been found in cases of Rieger's syndrome underscores the variance of phenotypic expression of this disorder (Alkemade, 1969). Microcornea, found bilaterally in the proband, is a relatively uncommon finding in Rieger's syndrome. Only $9 \%$ of eyes in Alkemade's series (1969) were found to have corneal diameters under $9.5 \mathrm{~mm}$. Actual corneal dimensions are often hard to determine accurately, as most cases of the disorder have indistinct limbi owing to persistent corneoscleral membranes. The proband had multiple iris abnormalities including stromal defects, hypoplasia of the anterior stroma, and ectropion uveae. The dyscoric, slit-like pupils are seen in most cases of Rieger's syndrome, though the large iridal defects, pseudopolycoria, such as those found in the proband are less common (Alkemade, 1969).

Most pedigrees of families with primary mesodermal dysgenesis show considerable interfamilial variability of phenotypic expression. The minimal ocular anomalies found in the proband's father, prominent iris mounds bilaterally, may represent a mild clinical manifestation of the disease. Burian (1957) reported a similar pedigree in which the father was found to have anterior chamber angle structures and irides which were interpreted as being abnormal only after stigmata of primary mesodermal dysgenesis were discovered in 4 of his 8 children.

Defects of the anterior chamber angle and iris such as colobomata and stromal hypoplasia are found in many of the autosomal syndromes. Hypoplasia of the iris is often seen in trisomy 21 in addition to the characteristic Brushfield spots (Lowe, 1949; Caginaut, 1968). Trisomy $13-15$ is notable for the extent of ocular involvement (Patau et al., 1960). These patients had the signs of generalised mesodermal dysplasia. Facial cleft defects and poor differentiation of the anterior chamber angle structures are common (Apple et al., 1970). The irides and choroid are usually very abnormal, often containing intraocular cartilage thought to be formed from mesodermal remnants within the ciliary body (Smith et al., 1963).

The ocular anomalies associated with trisomy 18 syndrome are largely external, but intraocular defects have been found (Edwards et al., 1960). Keith (1968) reviewed 5 cases and found microcornea, shallow anterior chambers, and abnormal ciliary processes in 1 case. Ginsberg et al. (1968) reported a case with eccentric pupils and another with iris sphincter atrophy, anomalous ciliary processes, and abnormal iris stroma.

Clinical syndromes involving chromosomal deletions also affect the iris and anterior chamber angle. Iris colobomata, sclerocornea, anterior chamber angle malformations, and ectopic pupils have been found in cases of $4 \mathrm{p}$ - (Wolf-Hirschorn) syndrome (François et al., 1972). Glaucoma is a prominent feature of 18q- (De Grouchy) syndrome and has been reported in association with anomalies of the anterior chamber angle (De Grouchy, 1965). Ring defects of chromosome 18 (18r syndrome) are found in patients with malformations of the anterior chamber angle and iris hypoplasia (Yanoff and Rorke, 1970).

One of us (Cotlier et al., 1977) presented a pedigree of partial trisomy $2 q$ syndrome of which 2 members had prominent, anteriorly displaced Schwalbe's line and iridocorneal adhesions. Although these patients lacked the iridal stromal hypoplasia and pupillary anomalies found in Rieger's syndrome, the abnormalities of the anterior chamber angle and the presence of glaucoma suggest a similar pathogenic mechanism.

The pathogenesis of Rieger's syndrome and similar ocular malformations has yet to be established. There are few animal models for the disease, though pedigrees of basenji dogs have been studied and found to have microcornea, persistent pupillary membranes, typical colobomata involving the iris, choroid, and retina, and sclerocorneal membranes (Barnett and Knight, 1969). One dog was also noted to have missing upper premolar teeth unilaterally. The mode of inheritance was autosomal dominant. Chromosomal analyses have not been reported.

The well-documented hereditary patterns of inheritance of Rieger's syndrome and the association of chromosomal defects with aberrant development of the anterior chamber angle and iris suggest a genetically-induced defect during early morphogenesis involving the iridogenic mesoderm, probably during the 14 to $20 \mathrm{~mm}$ state of development. The abnormalities of the iris, pupil, and anterior chamber angle are the result of imperfect progression of the cleavage process which forms the anterior chamber. No specific chromosome can be implicated, but the genetic imbalance resulting from a chromosomal defect may be enough to upset the delicate morphogenetic process.

This case represents only the second time that a chromosomal anomaly has been associated with Rieger's syndrome. The case of Tabbara et al. (1973) showed ocular and systemic defects in association with an extra metacentric chromosome, similar to a G-chromosome, and a missing group $\mathrm{C}$-chromosome. Clinical findings included many defects also noted in the proposita such as bilateral defects of the anterior chamber angle structures, microcornea, dyscoria, pseudopolycoria, hypoplasia of the maxilla, and anodontia. Defects of chromosome 6 have been associated with 5 cases in which defects of the 
Table 1 Aberrations of chromosome 6 with iris and anterior chamber defects

\begin{tabular}{|c|c|c|}
\hline Author & Ocular anomalies & Chromosomal defect \\
\hline $\begin{array}{l}\text { Rhode and Catz, } \\
1964\end{array}$ & Ectopic pupils & 6q partial trisomy \\
\hline $\begin{array}{l}\text { Goddé-Jolly } \\
\text { et al., } 1970\end{array}$ & $\begin{array}{l}\text { Opaque membrane } \\
\text { over anterior } \\
\text { chamber angle } \\
\text { structures }\end{array}$ & $46 X X-1-C t(1 p-; C p-q+)$ \\
\hline $\begin{array}{l}\text { Tabbara et al., } \\
1973\end{array}$ & $\begin{array}{l}\begin{array}{r}\text { Mesodermal } \\
\text { dysgenesis }\end{array} \\
\text { Microcornea } \\
\text { Glaucoma }\end{array}$ & $\begin{array}{l}\text { Presumptive isochromosome } \\
\text { of long arm of chromo- } \\
\text { some } 6\end{array}$ \\
\hline $\begin{array}{l}\text { Lamarr et al., } \\
\qquad 1974\end{array}$ & $\begin{array}{l}\text { Obstruction of } \\
\text { anterior chamber } \\
\text { angle by membrane } \\
\text { Processes extending } \\
\text { from membrane } \\
\text { to iris }\end{array}$ & $t(1 ; 6) q 23 ; q 27)$ \\
\hline $\begin{array}{c}\text { Tinning et al., } \\
1975\end{array}$ & Glaucoma & $t(1 ; 6)$ \\
\hline
\end{tabular}

anterior ocular segment and glaucoma were found (Table 1).

Supported by grants EY-00785 and EY-02490.

\section{References}

Alkemade, P. P. H. (1969). Dysgenesis mesodermalis of the iris and the cornea. A study of Rieger's Syndrome and Peter's Anomaly. Royal van Gorcum: The Netherlands.

Apple, D. J., Holden, J. D., and Stallworth, B. (1970). Ocular pathology of Patau's syndrome with an unbalanced D/D translocation. American Journal of Ophthalmology, 70, 383-391.

Barnett, K. C., and Knight, G. C. (1969). Persistent pupillary membrane and associated defects in the Basenji. Veterinary Record, 85, 242-248.

Burian, H. M., Rice, M. H., and Allen, L. (1957). External visibility of the region of Schlemm's canal. (Report on a family with developmental anomalies of cornea, iris, and chamber angle.) Archives of Ophthalmology, 57, 651-658.

Caginaut, B. (1968). Augenbefunde bei chromosomen krankheiten. Ophthalmologica, 155, 146-166.

Cotlier, E., Reinglass, H., and Rosenthal, I. (1977). The eye in partial trisomy 2q syndrome. American Journal of Ophthalmology, 84, 251-258.

DeGrouchy, J. (1965). Chromosome 18: a topologic approach. Journal of Pediatrics, 66, 414-431.
Edwards, J. H., Harnden, D. G., Cameron, A. H., Crosse, V. M., and Wolff, O. H. (1960). A new trisomic syndrome, Lancet, 1, 787-790.

François, J., Berger, R., and Saraux, H. (1972). Les aberrations chromosomiques en ophthalmologie. Masson: Paris.

Friedrich, U., and Nielson, J. (1973). Chromosome studies in five cases of coloboma of the iris. Lancet, 1, 576-578.

Ginsberg, J., Perrin, E. V., and Sueoka, W. T. (1968). Ocular manifestations of trisomy 18. American Journal of Ophthalmology, 66, 59-67.

Goddé-Jolly, D., Bonnefant, F., Raoul, O., Clergue, G. Mallet, R., and Leverne, J. (1970). A propos d'un cas de glaucome congenital associe a une anomalie chromosomique. Bulletin des Societes d'Ophthalmologie de France (Paris), 70, 875-881.

Jay, M. (1977). The eye in chromosome duplications and deficiencies. Marcel Dekker: New York.

Keith, C. G. (1968). The occular findings in the trisomy syndromes. Proceedings of the Royal Society of Medicine, 61, 251-253.

Lamarr, L. U., Friedrich, U., Peterson, G., Jorgenson, J., Nielson, J., Therkelsen, A. J., and Kissmeyer-Nielson, F. (1974). Assignment of a major histocompatibility complex to chromosome no. 6 in a family with pericentric inversion. Human Hereditary, 24, 273-284.

Lele, K. P., Dent, T., and Delhanty, J. D. A. (1965). Chromosome studies in five cases of coloboma of the iris. Lancet, 1, 576-578.

Lowe, R. F. (1949). The eyes in mongolism. British Journal of Ophthalmology, 33, 131-174.

Mikelsaar, A. V. N., Ananjev, E. V., and Gindilis, V. M. (1970). Probable pericentric inversion in chromosome No. 1 in a female child (46, XX, inv $1 \mathrm{p}+\mathrm{q}-)$. Human-genetik, 9, 316-324.

Patau, K., Smith, D. W., Therman, E., Inhorn, S. L., and Wagner, H. P. (1960). Multiple congenital anomaly caused by an extra autosome. Lancet, 1, 790-792.

Pitt, D. B., Weiner, S., Sutherland, G., and Pearch, P. (1967). The pericentric syndrome. Lancet, $2,568$.

Rhode, R. A., and Catz, B. (1964). Maternal transmission of a new group C (6/9) chromosomal syndrome. Lancet, 2 , 838-840.

Schmid, W. (1967). Pericentric inversions. Journal de genetique humaine, 16, 89-96.

Smith, D. W., Patau, K., Therman, E., Inhorn, S. I., and DeMars, R. I. (1963). The $D_{1}$ trisomy syndrome. Journal of Pediatrics, 62, 326-341.

Tabbara, K. F., Khouri, F. P., and DerKaloustian, V. M. (1973). Rieger's syndrome with chromosomal anomaly. Canadian Journal of Ophthalmology, 8, 488-491.

Tinning, S., Jacobsen, P., and Mikkelsen, M. (1975). A 1,6, translocation associated with congenital glaucoma and cleft lip and palate. Human Hereditary, 25, 453-460.

Waring, G. O., Rodrigues, M. M., and Laibson, P. R. (1975). Anterior chamber cleavage syndrome. A stepladder classification. Survey of Ophthalmology, 20, 3-27.

Yanoff, M., and Rorke, B. S. (1970). Ocular and cerebral abnormalities in chromosome 18 deletion defect. American Journal of Ophthalmologv, 70, 391-402. 